\title{
Local aspects of sleep and wakefulness
}

\author{
Francesca Siclari $^{1,2}$ and Giulio Tononi ${ }^{2}$
}

${ }^{1}$ Center for Research and Investigation on Sleep (CIRS), Lausanne University Hospital (CHUV) and University of Lausanne, Rue du Bugnon 46, 1011 Lausanne, Switzerland ${ }^{2}$ Department of Psychiatry, University of Wisconsin-Madison, 6001 Research Park Boulevard Madison, WI 53519, USA

\section{Abstract}

Slow waves, the hallmark of NREM (Non Rapid Eye Movement) sleep, are not uniformly distributed across the cortical surface, but can occur locally and asynchronously across brain regions. Their regional distribution and amplitude is affected by brain maturation and by time spent awake, mediated in part by experience-dependent changes in synaptic strength. Recent studies have shown that local low-frequency oscillations $(<10 \mathrm{~Hz})$ can also occur in REM sleep and during wakefulness, leading to region-specific cognitive errors. Local decreases and increases of slow wave activity in posterior brain regions have been linked to the occurrence of dreaming and to unconscious sleep, respectively. Finally, the coexistence of local sleep-like and wake-like patterns in different brain areas is characteristic of several sleep disorders and may offer insights into these conditions.

Sleep is typically considered a global phenomenon that affects the whole brain uniformly and simultaneously. Along the same lines, sleep and wakefulness are usually treated as two mutually exclusive behavioral states, with hardly any grey zone in between. This 'all-ornone view' of sleep has dominated sleep research for a long time, and is neatly reflected in the current classification of sleep stages, in which an unambiguous label of either sleep or wakefulness is attributed to each 30-s page of sleep recordings [1]. Undeniably, sleep and wakefulness are characterized by distinct behaviors, mental activity and responsiveness to the environment; they carry different EEG signatures and are influenced by specific neuromodulatory milieus [2]. Nonetheless, research in the last decades has started to refine this simplistic distinction and to address important questions: Can sleep occur and be regulated locally? Is it possible for sleep and wakefulness to co-exist in different areas of the brain? If so, what are the consequences on cognition and behavior? In the present article we will review local aspects of sleep and wakefulness, outline cognitive and behavioral correlates and discuss clinical implications, with an emphasis on recent findings. 


\section{The EEG of slow wave sleep}

For the purpose of this review, we will mostly focus on slow waves as the principal marker of Non-Rapid Eye Movement (NREM) sleep. Slow waves occur when neurons become bistable and undergo a slow oscillation in membrane potential between two states $(<1 \mathrm{~Hz})$ : a depolarized 'up-state' during which neurons fire ('on-period'), corresponding to the transition between the negative and the positive deflection of the EEG wave, and a hyperpolarized 'down-state' characterized by neuronal silence ('off-period'), which coincides with the positive to negative transition of the EEG wave [3]. Slow wave activity (SWA), which refers to the spectral power in the $0.5-4.5 \mathrm{~Hz}$ range, reflects both the amplitude and number of slow waves and is a reliable marker of the homeostatic regulation underlying sleep need: it is maximal at sleep onset, decreases progressively in the course of a night's sleep and increases with the duration of prior wakefulness [4].

\section{Local aspects of sleep}

Perhaps the most remarkable example of 'local' sleep is observed in some species of dolphins, like bottlenose dolphins, in which slow wave sleep can occur in only one hemisphere during swimming, while the other hemisphere displays low-voltage fast 'desynchronized' EEG activity typical of wakefulness [5]. Similar unihemispheric sleep patterns have been observed in several species of whales, manatees, some seals as well as several birds [6,7]. Although unihemispheric sleep does not occur in humans, a recent study has shown that during the first night in a laboratory environment, the left hemisphere displays less SWA and is more reactive to external stimuli compared to the right hemisphere, potentially acting as a 'night watch' to monitor unfamiliar surroundings [ $\left.8^{\circ}\right]$. In humans, SWA decreases along an anterior-posterior gradient, with frontal brain areas having the highest SWA in adults and the largest increase in SWA during recovery sleep after sleep deprivation $[9,10]$. Recently, intracranial recordings in epileptic patients have shown that during NREM sleep the alternation between off- and on-periods that underlies surface EEG slow waves is not global, but occurs mostly locally, meaning that it can be confined to specific brain regions and occur out-of-phase with respect to other cortical areas (Figure 1a) [11]. The simultaneous occurrence of slow waves and EEG activations in different brain regions is found even in the deepest stages of NREM sleep [12], but appears to be particularly marked towards the end of the sleep period [11]. These results indicate that slow waves characteristic of NREM sleep can occur in some brain regions while sparing others, suggesting that some aspects of sleep may be locally regulated [13]. Recent studies have a gone a step further by identifying local slow oscillations not only in NREM sleep, but also in REM sleep, which is traditionally characterized by an 'activated' or 'desynchronized' EEG similar to that of wakefulness. In particular, slow waves with neuronal off periods have been found in the middle and superficial layers of primary cortices in mice, and could account for sensory disconnection during this stage of sleep (Figure 1b) [14*0. Intriguingly, intracranial recordings in humans have also documented patterns of EEG activation during 'phasic' REM sleep (REM sleep with high density of eye movements) similar to those observed when performing a voluntary movement during wakefulness [15]. Altogether, these findings indicate that features thought to be exclusive of specific behavioral states, such as slow waves (NREM sleep), EEG activation (wakefulness, REM sleep) and EEG activity typical of 
voluntary movements (wakefulness), can be found, at a local level, also in other behavioral states.

\section{Local sleep and plasticity}

Local aspects of sleep can also be affected by prior experience and learning. In an early study, Huber et al. [16] showed that a motor learning task involving the right parietal region produced a local increase in SWA in this area during subsequent NREM sleep, compared to when the same task was performed without a learning component. Moreover, the local increase in SWA was correlated with task performance the next day and showed a homeostatic decline in the course of the first 90 min of sleep. Similar findings were obtained in rats [17]. By contrast, arm immobilization for $12 \mathrm{~h}$ during daytime produced a decrease in SWA over the sensorimotor cortex during subsequent NREM sleep, which was correlated with deterioration in motor performance [18]. More recent studies have documented regionally specific changes in the sleep EEG also for non-motor tasks. For instance, after prolonged audiobook listening, subjects displayed an increase in low-frequency EEG power during subsequent sleep in left frontal brain derivations whereas, after playing a driving simulator game, they showed SWA increases in right parietal areas (Figure 1c) [19². In another study, a visual perceptual learning task involving orientation-selective neurons in the lateral occipital cortex led to an increase in the number of slow waves initiated in this area [20].

The distribution of SWA is also influenced by developmental changes. In young children, the slow wave maximum is confined to occipital regions, and progressively shifts to anterior brain regions with age, in parallel with brain maturation and the development of regionally specific skills [21,22]. Overall, SWA shows a remarkable decline with age, particularly during adolescence [23]. A recent study using a large-scale neural model of primary visual cortex and sections of the thalamus showed that synaptic refinement, the reorganization of synapses and connections without significant change in their number or strength, could

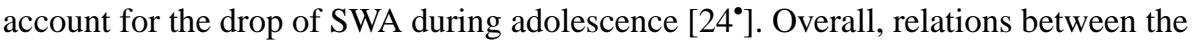
regional distribution of SWA and prior use and with maturational changes suggest that SWA may be modulated by plasticity-related processes. Indeed, several lines of evidence indicate that SWA is related to synaptic changes that ultimately account for the benefits of sleep including learning and memory consolidation $\left[25^{\circ}\right]$. A detailed outline of current theories on the topic is beyond the scope of this article and can be found in Refs. $\left[25^{\circ}, 26\right]$.

\section{Local sleep in wakefulness}

When individuals remain awake for extended periods of time, they become sleepy and show behavioral changes and cognitive impairment. Under conditions of sleep deprivation, the waking EEG typically shows increased low-frequency power in the theta range $(5-7 \mathrm{~Hz})$ reflecting the duration of prior wakefulness [27-29]. A recent study using local field potentials and multi-unit recordings has shown that when rats are kept awake beyond the usual duration, cortical neurons tend to fall silent for brief periods, as they do during NREM sleep [30]. These 'off periods' are associated with slow oscillations in the slow/theta range in LFP recordings, occur for the most part asynchronously across brain regions and increase with time spent awake. Importantly, while the animal appears behaviorally awake, the 
occurrence of off-periods over motor areas is associated with errors in a reaching task. A more recent study performed in humans showed that the increase in waking theta EEG activity during sleep deprivation displayed regional, task dependent changes. More specifically, it increased predominantly over left frontal brain regions after a language-based task and over posterior parietal regions after a visuomotor task. The same regions displayed local increases in low-frequency power including SWA during subsequent recovery sleep [19*]. In addition, the occurrence of theta waves in task-related regions coincides with specific performance errors in humans $\left[31^{\circ}\right]$. Taken together, these findings suggest that local sleep can occur while animals and humans appear awake and may account for at least some of the cognitive and behavioral manifestations of sleepiness.

\section{Local sleep in state transitions}

State transitions, in which sleep and wakefulness replace each other, provide an excellent opportunity to observe local sleep. Indeed, several recent studies have shown that the transition to sleep is not a spatially and temporally uniform process, but shows regionally specific features. In addition, recent work using refined techniques of signal analysis has started to shed light on how local sleep patterns relate to cognitive activities during state transitions. In the falling asleep period, SWA typically appears first in fronto-central regions [32,33], and only later in posterior brain areas. The relative sparing of sensory, parietooccipital regions by slow waves in the early part of the falling asleep process [33] may account for the highly perceptual experiences (hypnagogic hallucinations) that frequently occur at sleep onset. Indeed, the content of visual imagery at sleep onset can be successfully predicted based on brain activity in visual areas by using a decoding algorithm that has previously been trained on waking data during movie watching [34*0. This suggests that visual imagery at sleep onset and visual perception during wake share similar anatomical substrates. At the subcortical level, it was found that the thalamus undergoes deactivation before neocortical areas [35] during the transition to sleep. In addition, the hippocampus displays sleep spindles several minutes before sleep onset, and hippocampal spindle detections consistently precede neocortical events with increasing delays along the cortical antero-posterior axis [ $\left.36^{\circ}\right]$. The presence of hippocampal sleep spindles before the cortical EEG-defined sleep onset may explain the frequent amnesia for events that occur immediately 'before' falling asleep $\left[36^{\circ}, 37\right]$.

Spatially heterogeneous sleep-like EEG patterns have also been documented in the awakening process. Regional dissociations between cortical and subcortical structures can be seen using PET imaging: cerebral blood flow is most rapidly reestablished in centrencephalic regions (brainstem, thalamus) while in heteromodal neo-cortical areas, blood flow continues to increase up to 20 min later [38]. Compared to pre-sleep wakefulness, the first minutes after morning awakenings from both REM and NREM sleep are characterized by increased SWA in posterior brain regions $\left[39^{\circ}, 40\right]$. A recent study using intracortical recordings in rats and mice has identified distinct populations of cortical neurons that are virtually silent upon arousal, and only reach average waking levels within the next 5-10 min. Interestingly, the incidence of off periods was higher when animals were awoken from REM as compared to NREM sleep [41.']. 


\section{Local sleep and consciousness}

How does regionally heterogeneous SWA affect conscious experiences during sleep? It is now well established that dreaming can occur not only in REM sleep, but also in NREM sleep. Particularly at the end of the night, when SWA is reduced, dream reports from NREM and REM sleep become virtually indistinguishable, suggesting that slow waves may interfere with consciousness [42]. Several lines of evidence suggest that this is indeed so. Earlier studies using transcranial magnetic stimulation (TMS) have shown that the bistability of thalamocortical neurons during NREM sleep causes a breakdown of effective connectivity among specialized thalamocortical modules [43,44], thereby impairing information integration, a theoretical prerequisite for consciousness $\left[45^{\circ}\right]$. Indeed, a recent study using intracranial recordings in humans has shown that the neuronal off periods associated with slow waves interrupt the 'causal chain' between periods of activation [46"*]. More specifically, electrical stimulation of the cortex in NREM sleep induces a slow wave associated with an OFF-period (neuronal silence), after which neuronal activity resumed to wakefulness-like levels. However, the causal effect of the initial stimulation on this activity, measured by phase-locking, is lost, indicating an interruption of deterministic interactions. Another recent study showed that, within NREM sleep, TMS evokes a larger negative deflection and a shorter phase-locked response when subjects reported unconsciousness compared to when they reported dreaming $\left[47^{\circ}\right]$, and that the amplitude of the negative deflection, a marker of bistability, was negatively correlated with the length of the dream report. Early attempts to relate spontaneous EEG power changes to changes in consciousness have yielded variable results, although a few suggested that low-frequency EEG power is negatively associated with mental experiences during sleep [48,49]. A recent large-scale study using refined techniques of signal analysis (high-density EEG recordings with source reconstruction), comprising over 30 subjects and more than 1000 awakenings, showed that reports of conscious experiences are preceded by less SWA compared to reports of unconsciousness $\left[50^{\circ}\right]$. Intriguingly, this reduction of SWA was confined to a parietooccipital 'hot zone' in both REM and NREM sleep, comprising the medial and lateral occipital lobe, extending superiorly to the precuneus and posterior cingulate gyrus. Monitoring this posterior hot zone in real time predicted whether an individual would report dreaming or the absence of experiences during NREM sleep. These results suggest that conscious experiences during sleep can occur when there is a localized reduction of SWA in a posterior hot zone, irrespective of the EEG in the rest of the cortex. Thus, the absence or presence of local sleep slow waves in this posterior hot zone may explain why dreaming as well as unconsciousness can occur in both REM and NREM sleep despite the radically different globalEEG patterns of these two states.

\section{Local sleep and sleep disorders}

The study of the mechanisms underlying local sleep patterns has direct implications for clinical conditions. Indeed, several sleep disorders are characterized by a so-called 'state dissociation', in which behavioral features of more than one state coexist [51]. Sleepwalking and other disorders of arousal, for instance, result from incomplete awakenings during slow wave sleep, and are characterized by impaired cognition, different degrees of retrograde amnesia and variable motor activity. Several earlier studies had shown that during such 
episodes, localized wake-and sleep like patterns coexist in different brain areas [52-54]. A more recent study that took advantage of the increased spatial resolution of high-density EEG recordings showed that, compared to healthy controls, sleepwalkers display reduced SWA at the level of the cingulate, motor and sensorimotor associative cortex across

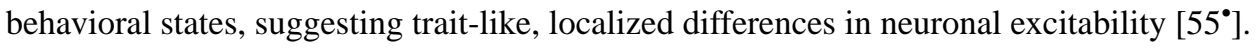
Similarly, EEG recordings in subjects with insomnia show locally increased wake-like activity in sensorimotor areas during sleep as compared to healthy controls [ $\left.56^{\circ}\right]$. Finally, patients with narcolepsy display a wide range of clinical phenomena related to state dissociation [51], including excessive daytime sleepiness, sleep paralysis, sleep-related hallucinations, cataplexy and lucid dreaming [57]. In the future, a systematic assessment of local sleep features in affected patients will help outline the neuroanatomical circuits implicated in the pathogenesis of these conditions and offer further insight into the behavioral and cognitive correlates of local sleep.

\section{Conclusion}

It is now well established that sleep occurs and is regulated locally. Recent studies have shown that local aspects of sleep, such as low-frequency oscillations typical of NREM sleep, can also occur during extended periods of wakefulness and even during REM sleep. The occur-rence of such local low-frequency oscillations is in part modulated by prior experience and learning, and may account for cognitive impairment and regionally specific performance errors during sleep deprivation in both humans and animals. The occurrence of local slow wave activity in posterior brain regions may also explain whether one is unconscious or dreaming. Finally, local dissociations between different behavioral states are a feature of several sleep disorders.

\section{Acknowledgments}

Funding

This work was supported by the Swiss National Science Foundation Grants139778 (FS), 145571 (FS), the Swiss Foundation for Medical Biological Grants151743 and 145763 (FS), the Divesa foundation Switzerland (FS), the Pierre-Mercier foundation for Science (FS), the bourse pro-femmes of the CHUV and University of Lausanne, Switzerland (FS), NIH/NIMH grant R01MH099231 (GT), NIH/NINDS grant P01NS083514 (GT), and NIH/ NCCAMP01AT004952 (GT)

\section{References and recommended reading}

Papers of particular interest, published within the period of review, have been highlighted as:

- of special interest

•• of outstanding interest

1. Iber C, Ancoli-Israel S, Chesson A, Quan SF: The AASM Manual for the Scoring of Sleep and Associated Events: Rules, Terminology and Technical Specifications. 1st edn Westchester, Illinois: American Academy of Sleep Medicine; 2007.Study using intracranial recordings to outline regionally specific onsets of spindles in the falling asleep period.

2. Jones BE: From waking to sleeping: neuronal and chemical substrates. Trends Pharmacol. Sci 2005, 26:578-586. [PubMed: 16183137] 
3. Steriade M, Timofeev I, Grenier F: Natural waking and sleep states: a view from inside neocortical neurons. J. Neurophysiol 2001, 85:1969-1985. [PubMed: 11353014]

4. Achermann P, Borbely AA: Mathematical models of sleep regulation. Front. Biosci 2003, 8:s683693. [PubMed: 12700054]

5. Mukhametov LM, Supin AY, Polyakova IG: Interhemispheric asymmetry of the electroencephalographic sleep patterns in dolphins. Brain Res 1977, 134:581-584. [PubMed: 902119]

6. Mascetti GG: Unihemispheric sleep and asymmetrical sleep: behavioral, neurophysiological, and functional perspectives. Nat. Sci. Sleep 2016, 8:221-238. [PubMed: 27471418]

7. Lesku JA, Vyssotski AL, Martinez-Gonzalez D, Wilzeck C, Rattenborg NC: Local sleep homeostasis in the avian brain: convergence of sleep function in mammals and birds? Proc. Biol. Sci 2011, 278:2419-2428. [PubMed: 21208955]

8•. Tamaki M, Bang JW, Watanabe T, Sasaki Y: Night watch in one brain hemisphere during sleep associated with the first-night effect in humans. Curr. Biol 2016, 26:1190-1194. [PubMed: 27112296] Study showing hemispheric asymetries in slow wave activity and brain reactivity during a first night in an unfamiliar laboratory environment.

9. Finelli LA, Borbely AA, Achermann P: Functional topography of the human nonREM sleep electroencephalogram. Eur. J. Neurosci 2001, 13:2282-2290. [PubMed: 11454032]

10. Werth E, Achermann P, Borbely AA: Fronto-occipital EEG power gradients in human sleep. J. Sleep Res. 1997, 6:102-112. [PubMed: 9377529]

11. Nir Y, Staba RJ, Andrillon T, Vyazovskiy VV, Cirelli C, Fried I, Tononi G: Regional slow waves and spindles in human sleep. Neuron 2011, 70:153-169. [PubMed: 21482364]

12. Nobili L, Ferrara M, Moroni F, De Gennaro L, Russo GL, Campus C, Cardinale F, De Carli F: Dissociated wake-like and sleep-like electro-cortical activity during sleep. Neuroimage 2011, 58:612-619. [PubMed: 21718789]

13. Krueger JM, Tononi G: Local use-dependent sleep; synthesis of the new paradigm. Curr. Top. Med. Chem 2011, 11:2490-2492. [PubMed: 21906015]

14••. Funk CM, Honjoh S, Rodriguez AV, Cirelli C, Tononi G: Local slow waves in superficial layers of primary cortical areas during REM sleep. Curr. Biol 2016, 26:396-403. [PubMed: 26804554] Study documenting slow waves with neuronal off-periods in REM sleep.

15. De Carli F, Proserpio P, Morrone E, Sartori I, Ferrara M, Gibbs SA, De Gennaro L, Lo Russo G, Nobili L: Activation of the motor cortex during phasic rapid eye movement sleep. Ann. Neurol 2016, 79:326-330. [PubMed: 26575212]

16. Huber R, Ghilardi MF, Massimini M, Tononi G: Local sleep and learning. Nature 2004, 430:7881. [PubMed: 15184907]

17. Hanlon EC, Faraguna U, Vyazovskiy VV, Tononi G, Cirelli C: Effects of skilled training on sleep slow wave activity and cortical gene expression in the rat. Sleep 2009, 32:719-729. [PubMed: 19544747]

18. Huber R, Ghilardi MF, Massimini M, Ferrarelli F, Riedner BA, Peterson MJ, Tononi G: Arm immobilization causes cortical plastic changes and locally decreases sleep slow wave activity. Nat. Neurosci 2006, 9:1169-1176. [PubMed: 16936722]

19•. Hung CS, Sarasso S, Ferrarelli F, Riedner B, Ghilardi MF, Cirelli C, Tononi G: Local experiencedependent changes in the wake EEG after prolonged wakefulness. Sleep 2013, 36:59-72. [PubMed: 23288972] Study demonstrating local, task-dependent increases in theta activity after extended periods of wakefulness in humans.

20. Mascetti L, Muto V, Matarazzo L, Foret A, Ziegler E, Albouy G, Sterpenich V, Schmidt C, Degueldre C, Leclercq Y et al.: The impact of visual perceptual learning on sleep and local slowwave initiation. J. Neurosci 2013, 33:3323-3331. [PubMed: 23426660]

21. Kurth S, Ringli M, Geiger A, LeBourgeois M, Jenni OG, Huber R: Mapping of cortical activity in the first two decades of life: a high-density sleep electroencephalogram study. J. Neurosci 2010, 30:13211-13219. [PubMed: 20926647]

22. Kurth S, Ringli M, Lebourgeois MK, Geiger A, Buchmann A, Jenni OG, Huber R: Mapping the electrophysiological marker of sleep depth reveals skill maturation in children and adolescents. Neuroimage 2012, 63:959-965. [PubMed: 22498654] 
23. Feinberg I, Campbell IG: Sleep EEG changes during adolescence: an index of a fundamental brain reorganization. Brain Cogn. 2010, 72:56-65. [PubMed: 19883968]

24•. Hoel EP, Albantakis L, Cirelli C, Tononi G: Synaptic refinement during development and its effect on slow-wave activity: a computational study. J. Neurophysiol 2016, 115:2199-2213. [PubMed: 26843602] Study using a large-scale neural model showing that synaptic refinement could account for the drop of SWA during adolescence [22].

25•. Tononi G, Cirelli C: Sleep and the price of plasticity: from synaptic and cellular homeostasis to memory consolidation and integration. Neuron 2014, 81:12-34. [PubMed: 24411729] Recent comprehensive review of the synaptic homeostatis hypothesis, which links local sleep to plasticity and synaptic changes.

26. Born J, Rasch B, Gais S: Sleep to remember. Neuroscientist 2006, 12:410-424. [PubMed: 16957003]

27. Leemburg S, Vyazovskiy VV, Olcese U, Bassetti CL, Tononi G, Cirelli C: Sleep homeostasis in the rat is preserved during chronic sleep restriction. Proc. Natl. Acad. Sci. U. S. A 2010, 107:1593915944. [PubMed: 20696898]

28. Finelli LA, Baumann H, Borbely AA, Achermann P: Dual electroencephalogram markers of human sleep homeostasis: correlation between theta activity in waking and slow-wave activity in sleep. Neuroscience 2000, 101:523-529. [PubMed: 11113301]

29. Vyazovskiy VV, Tobler I: Theta activity in the waking EEG is a marker of sleep propensity in the rat. Brain Res. 2005, 1050:64-71. [PubMed: 15975563]

30. Vyazovskiy VV, Olcese U, Hanlon EC, Nir Y, Cirelli C, Tononi G: Local sleep in awake rats. Nature 2011, 472:443-447. [PubMed: 21525926]

31 . Bernardi G, Siclari F, Yu X, Zennig C, Bellesi M, Ricciardi E, Cirelli C, Ghilardi MF, Pietrini P, Tononi G: Neural and behavioral correlates of extended training during sleep deprivation in humans: evidence for local, task-specific effects. J. Neurosci 2015, 35:4487-4500. [PubMed: 25788668] Study showing that the occurrence of theta waves during sleep deprivation coincides with regionally and task-specific performance errors in humans.

32. Marzano C, Moroni F, Gorgoni M, Nobili L, Ferrara M, De Gennaro L: How we fall asleep: regional and temporal differences in electroencephalographic synchronization at sleep onset. Sleep Med. 2013, 14:1112-1122. [PubMed: 24051119]

33. Siclari F, Bernardi G, Riedner B, LaRocque J, Benca MR, Tononi G: Two distinct synchronization processes in the transition to sleep: a high-density electroencephalographic study. Sleep 2014, 37:1621-1637. [PubMed: 25197810]

34••. Horikawa T, Tamaki M, Miyawaki Y, Kamitani Y: Neural decoding of visual imagery during sleep. Science 2013, 340:639-642. [PubMed: 23558170] Study decoding the content of visual experiences from fMRI activity in visual areas at sleep onset, using a classifier that was trained on waking data while subjects were watching movies.

35. Magnin M, Rey M, Bastuji H, Guillemant P, Mauguiere F, Garcia-Larrea L: Thalamic deactivation at sleep onset precedes that of the cerebral cortex in humans. Proc. Natl. Acad. Sci. U. S. A 2010, 107:3829-3833. [PubMed: 20142493]

36• Sarasso S, Proserpio P, Pigorini A, Moroni F, Ferrara M, De Gennaro L, De Carli F, Russo GL, Massimini M, Nobili L: Hippocampal sleep spindles preceding neocortical sleep onset in humans. Neuroimage 2014, 86:425-432. [PubMed: 24176868]

37. Wyatt JK, Bootzin RR, Anthony J, Bazant S: Sleep onset is associated with retrograde and anterograde amnesia. Sleep 1994, 17:502-511. [PubMed: 7809563]

38. Balkin TJ, Braun AR, Wesensten NJ, Jeffries K, Varga M, Baldwin P, Belenky G, Herscovitch P: The process of awakening: a PET study of regional brain activity patterns mediating the reestablishment of alertness and consciousness. Brain 2002, 125:2308-2319. [PubMed: 12244087]

39•. Marzano C, Ferrara M, Moroni F, De Gennaro L: Electroencephalographic sleep inertia of the awakening brain. Neuroscience 2011, 176:308-317. [PubMed: 21167917]

40. Ferrara M, Curcio G, Fratello F, Moroni F, Marzano C, Pellicciari MC, Gennaro LD: The electroencephalographic substratum of the awakening. Behav. Brain Res 2006, 167:237-244. [PubMed: 16242789] 
41•. Vyazovskiy VV, Cui N, Rodriguez AV, Funk C, Cirelli C, Tononi G: The dynamics of cortical neuronal activity in the first minutes after spontaneous awakening in rats and mice. Sleep 2014, 37:1337-1347. [PubMed: 25083014] Study showing the dynamics of off-periods in cortical neurons after awakenings from both NREM and REM sleep.

42. Nir Y, Tononi G: Dreaming and the brain: from phenomenology to neurophysiology. Trends Cogn. Sci 2010, 14:88-100. [PubMed: 20079677]

43. Massimini M, Ferrarelli F, Huber R, Esser SK, Singh H, Tononi G: Breakdown of cortical effective connectivity during sleep. Science 2005, 309:2228-2232. [PubMed: 16195466]

44. Massimini M, Ferrarelli F, Murphy M, Huber R, Riedner B, Casarotto S, Tononi G: Cortical reactivity and effective connectivity during REM sleep in humans. Cogn. Neurosci. 2010, 1:176183.

45• Tononi G: Consciousness as integrated information: a provisional manifesto. Biol. Bull 2008, 215:216-242. [PubMed: 19098144]

46••. Pigorini A, Sarasso S, Proserpio P, Szymanski C, Arnulfo G, Casarotto S, Fecchio M, Rosanova M, Mariotti M, Lo Russo G et al.: Bistability breaks-off deterministic responses to intracortical stimulation during non-REM sleep. Neuroimage 2015, 112:105-113. [PubMed: 25747918] Study showing that neuronal off-periods in humans break 'the causal chain' between neuronal activations, which may explain why consciousness fades in early slow wave sleep.

47• Nieminen JO, Gosseries O, Massimini M, Saad E, Sheldon AD, Boly M, Siclari F, Postle BR, Tononi G: Consciousness and cortical responsiveness: a within-state study during non-rapid eye movement sleep. Sci. Rep 2016, 6:30932. [PubMed: 27491799] Study showing that the amplitude of the slow-wave like response induced by TMS during NREM sleep can be used to distinguish reports of unconsciousness from reports of consciousness including dreaming.

48. Chellappa SL, Frey S, Knoblauch V, Cajochen C: Cortical activation patterns herald successful dream recall after NREM and REM sleep. Biol. Psychol 2011, 87:251-256. [PubMed: 21419827]

49. Esposito MJ, Nielsen TA, Paquette T: Reduced Alpha power associated with the recall of mentation from Stage 2 and Stage REM sleep. Psychophysiology 2004, 41:288-297. [PubMed: 15032994]

50•. Siclari F, Baird B, Perogamvros L, Bernardi G, LaRocque J, Riedner B, Boly M, Postle BR, Tononi G: The neural correlates of dreaming. Nat. Neurosci 2017 10.1038/nn.4545.epub ahead of print. Study showing that dreaming is associated with local SWA decreases in a parieto-occipital 'hot zone' of the brain, while unconsciousness during sleep is associated with SWA increases in the same area.

51. Mahowald MW, Schenck CH: Dissociated states of wakefulness and sleep. Neurology 1992, 42:44-51 discussion 52.

52. Bassetti C, Vella S, Donati F, Wielepp P, Weder B: SPECT during sleepwalking. Lancet 2000, 356:484-485. [PubMed: 10981896]

53. Terzaghi M, Sartori I, Tassi L, Didato G, Rustioni V, LoRusso G, Manni R, Nobili L: Evidence of dissociated arousal states during NREM parasomnia from an intracerebral neurophysiological study. Sleep 2009, 32:409-412. [PubMed: 19294961]

54. Terzaghi M, Sartori I, Tassi L, Rustioni V, Proserpio P, Lorusso G, Manni R, Nobili L: Dissociated local arousal states underlying essential clinical features of non-rapid eye movement arousal parasomnia: an intracerebral stereo-electroencephalographic study. J. Sleep Res 2012, 21:502-506. [PubMed: 22329925]

55•. Castelnovo A, Riedner BA, Smith RF, Tononi G, Boly M, Benca RM: Scalp and source power topography in sleepwalking and sleep terrors: a high-density EEG study. Sleep 2016, 39:18151825. [PubMed: 27568805] Recent study documenting a localized decrease in slow wave activity in subjects with arousal disorders as a trait-like factor.

56•. Riedner BA, Goldstein MR, Plante DT, Rumble ME, Ferrarelli F, Tononi G, Benca RM: Regional patterns of elevated alpha and high-frequency electroencephalographic activity during nonrapid eye movement sleep in chronic insomnia: a pilot study. Sleep 2016, 39:801-812. [PubMed: 26943465] Recent study documenting dissociated wake-sleep patterns in subjects with insomnia.

57. Dodet P, Chavez M, Leu-Semenescu S, Golmard JL, Arnulf I: Lucid dreaming in narcolepsy. Sleep 2015, 38:487-497. [PubMed: 25348131] 


\section{Local aspects of sleep}

(a) In NREM sleep

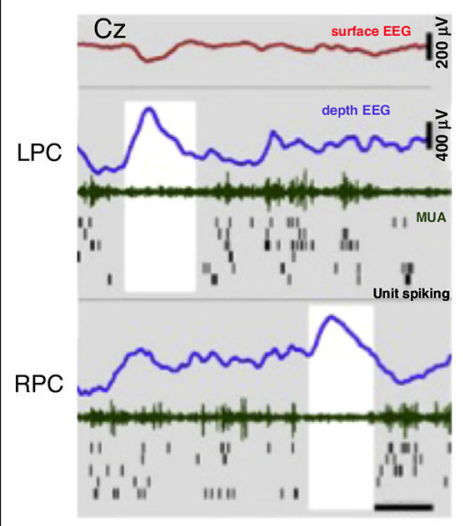

(b) In REM sleep

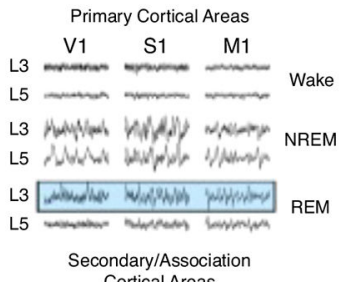

Secondary/Association
Cortical Areas

L3 V2 M2 RS

L5 Wake

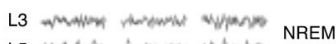

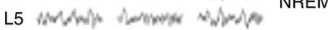

L3 memin

L5 (c) In wakefulness

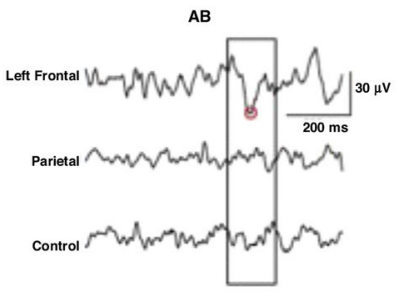

DS

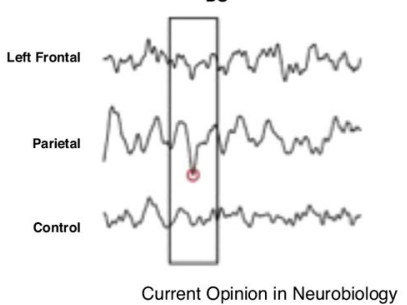

Figure 1.

Local aspects of sleep. (a) Example of local sleep slow waves in human NREM sleep occurring at different times in left and right posterior cingulate cortices, where $100 \%$ of units are locked to slow waves. Rows (top to bottom) depict activity in scalp EEG (Cz, red), left posterior cingulate, and right posterior cingulate. Blue, depth EEG; green, MUA; black lines, single-unit spikes. White shadings mark local OFF periods (reproduced, with permission, from Ref. [11]). (b) Laminar recordings revealing slow waves in layer 3 and 4 of mouse primary cortex in REM sleep (reproduced, with permission, from Ref. [14*0]). (c) Representative examples of local theta waves (boxed) occurring in left frontal derivations (top panel) after prolonged audio-book (AB) listening and in parietal derivations after playing with a driving simulator game for extended periods of time. Red circles indicate the negative peaks of theta waves detected in each EEG trace (reproduced, with permission, from Ref. [19]). 